\title{
Preface: Personalized Nanomedicine
}

Nanomedicine has contributed significantly to the progress of diagnostics and therapeutics for various diseases. With the rapid advancement of technology, nanomedicine-based biomedical applications progressed more toward personalized diagnostics and therapeutics. Development of multifunctional nanomaterials led to smart applications including image-guided therapy and theranostics, which combines diagnostic and therapeutic applications in a single system. Combined with the rapid growth of computational technology such as machine learning, personalized nanomedicine possesses huge potential for individually tailored and highly precise diagnostics and therapy.

This special issue of Critical Reviews ${ }^{\mathrm{TM}}$ in Biomedical Engineering highlights the topics presented at the 6th Personalized NanoMedicine Symposium, which was held on November 20, 2019 on the campus of Florida International University in Miami, Florida. The Society for Personalized Nano-Medicine (SPNM) aims to tailor medical intervention to patient- and disease- specific needs. Founded in 2013 as a non-for-profit organization, the mission of the SPNM is to promote research, serve as a source of information on current applications of nanotechnology, and foster exchange of information and ideas on personalized nanomedicine. Our vision and goal are to bring cross-disciplinary research to come together as a society to expand our understanding of current applications of nanotechnology. This symposium was designed for basic, translational, and clinical researchers to share the latest studies, important developments, and best research methods in the growing fields of nanomedicine and nanotechnology. The scientific program of this Symposium included the following topics in the fields of nanomedicine and personalized medicine:

1. Combinatory Therapeutic Strategies to Cure HIV

2. Biosensors: From Cell Recording to Point of Care Testings

3. Design and Development of Nanoparticles/ Formulations for Theranostics
4. Cryptic Peptidome, Opioid Addictions and E-cigarettes: A Potential Role in Nanomedicine

This special issue comprises written contributions of the presentations during SPNM including three review articles and two original research articles. The review articles summarize the recent advances and challenges of nanomedicine in brain diseases, lung diseases, and cancer treatment. Prof. Chand overviews the role of mitochondria in lung inflammation and Alzheimer's disease and the advancement of mitochondria-targeting nanotherapy. Prof. Unwalla describes the potential of CRISPR-Cas 9 technology in airway diseases. The third review, contributed by Prof. Raymond, summarizes the potential of bioengineered exosomes for cancer nanotherapy. Prof. Mahajan contributed an original research article focused on Alzheimer's disease theranostics using curcumin-pluronic nanoparticles. Prof. Raymond's group developed magnetic nanoparticle and exosome coupled nanocarriers for HIV therapeutics.

We would like to express our gratitude to all the authors for their remarkable contributions to this special issue. We believe that all the papers published in this special issue will have great influence in the field of personalized nanomedicine.

\section{Guest Editors:}

Asahi Tomitaka

Department of Immunology and Nano-Medicine

Institute of NeuroImmune Pharmacology, Centre for Personalized Nanomedicine

Herbert Wertheim College of Medicine

Florida International University, Miami, Florida 33199, USA

Chen-Zhong Li

Department of Biomedical Engineering

Florida International University, 10555 West Flagler

Street, Miami, FL 33174, USA 
Madhavan Nair

Department of Immunology and Nano-Medicine Institute of NeuroImmune Pharmacology, Centre for Personalized Nanomedicine

Dr. Asahi Tomitaka is an Assistant Professor in the Department of Immunology and Nano-Medicine at Herbert Wertheim College of Medicine, Florida International University. She received her Ph.D. in Electrical and Computer Engineering from Yokohama National University, Yokohama, Japan in 2011. Her research interests lie in the area of nanobiotechnology and magnetic engineering. Her current research focuses on image-guided drug delivery and theranostics using multifunctional nanoparticles. She has had postdoctoral training in the fields of biomaterials, materials science, and

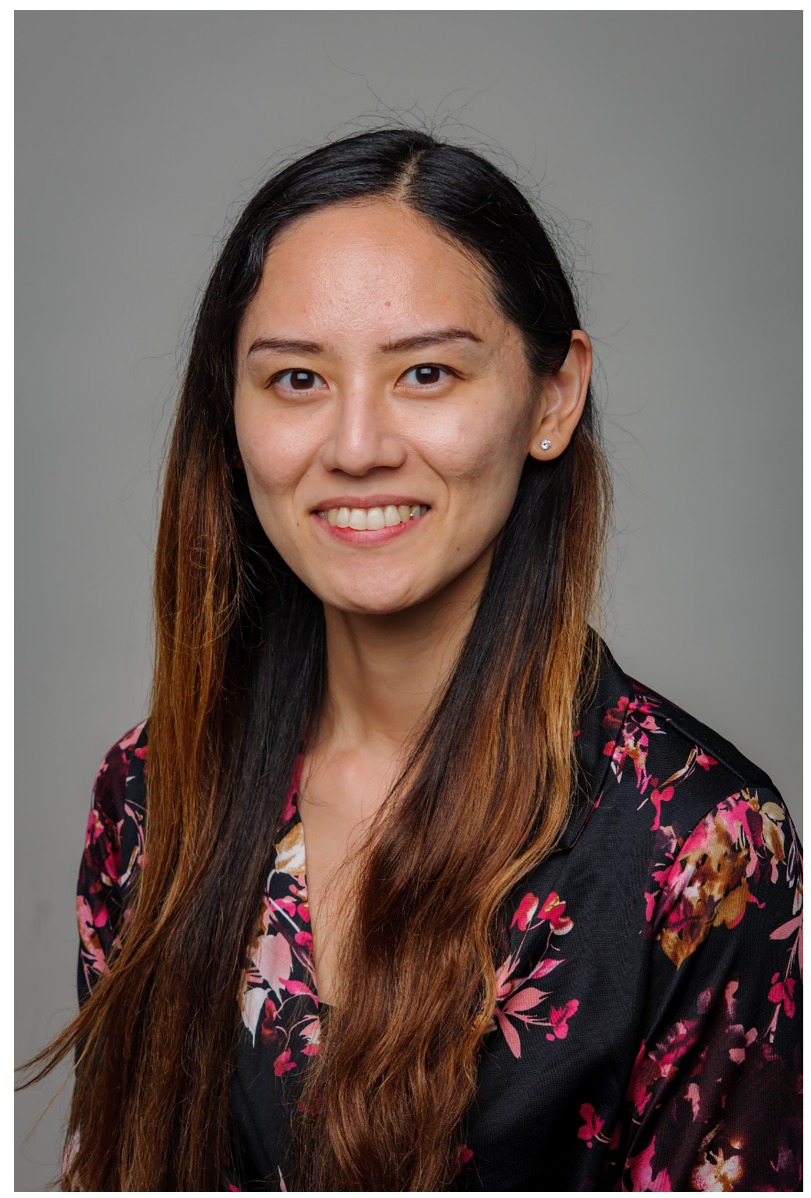

Herbert Wertheim College of

Medicine

Florida International University, Miami, Florida 33199, USA

nanomedicine. During her Ph.D. and postdoctoral training, she was awarded the Japan Society for the Promotion of Science (JSPS) Research Fellowship and JSPS Postdoctoral Fellowship for Research Abroad.

Prof. Chen-zhong Li is Professor of Biomedical Engineering and Director of the Nanobioengineering/Bioelectronics Lab at Florida International University (FIU), and the former program director (2017-2019) of the Biosensing program of the National Science Foundation. Dr. Li's research interests include biosensors, in vitro diagnosis, point care of testing, neuron-device interface, cell/organ on a chip, electric therapy, and electron transfer study of various biomaterials.

The impact of Dr. Li's research has been documented in 10 granted patents, approximately 140

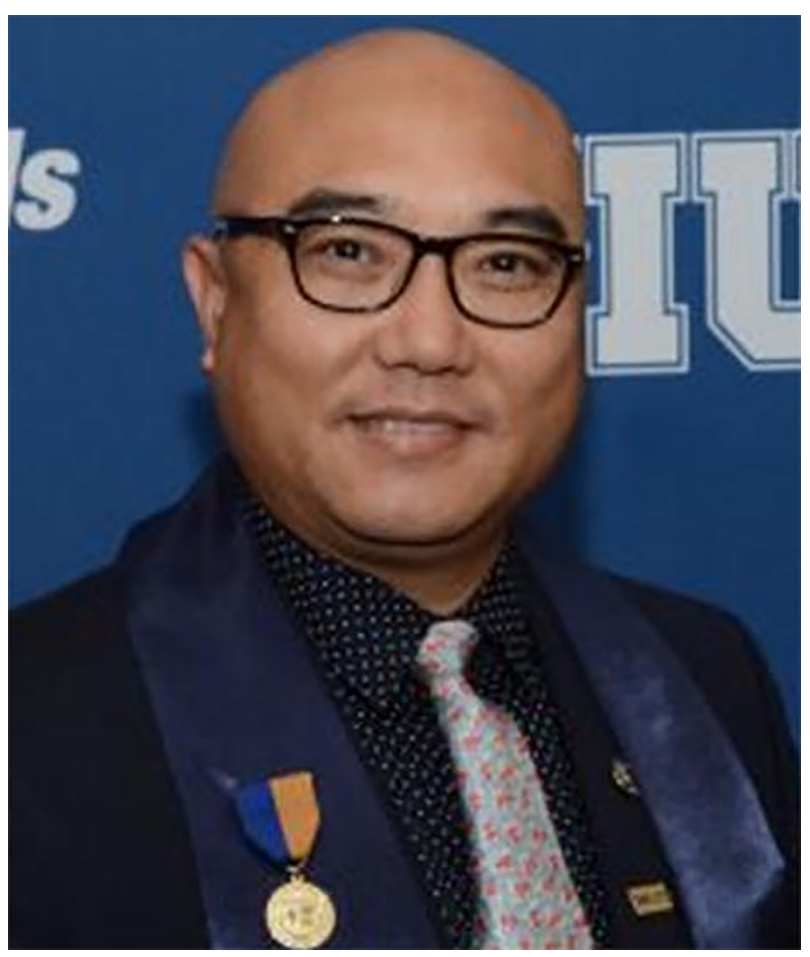


peer-reviewed journal papers, 2 books, 7 book chapters, and more than 120 keynote/invited lectures and seminars at national/international conferences and institutions. Dr. Li is the Co-Editor-in-Chief of the flagship journal Biosensors and Bioelectronics, the former Editor-in-Chief of the journal Critical Reviews $^{\mathrm{TM}}$ in Biomedical Engineering, and the associated editor of several other journals including Microsystems \& Nanoengineering, among others.

Additionally, he is an elected fellow of the American Institute for Medical and Biological Engineering (AIMBE) and a senior member of IEEE. In recognition for his work, Dr. Li has received several awards and honors including the Kauffman Entrepreneurship Professor Award in 2009 and 2011, 2014 JSPS (Japan) Professor Fellowship Award, 2014 FIU Excellent Faculty Award in Research and Creative Activities, 2016 Pioneer in Technology Development Award by the Society of Braining Mapping \& Therapeutics and Brain Mapping Foundations, 2016 Finalist for FIU President's Council Worlds Ahead Award, and 2016 Minority-Serving Institution Faculty Award in Cancer Research, by the American Association for Cancer Research.

Madhavan Nair, $\mathrm{PhD}$ received his $\mathrm{PhD}$ from Tata Memorial Cancer Center, Bombay University, India in Cancer Immunology and trained at Memorial Sloan Kettering Cancer Center, New York City. He then joined the faculty of the Department of Pediatrics at University of Michigan, Ann Arbor, and subsequently worked at the Department of Medicine and Microbiology at SUNY, Buffalo, NY as a Tenured Professor and Director of Research in Allergy and Immunology. He is a certified Clinical Nutrition Specialist (CNS), Fellow of the American College of Nutrition (FACN), Fellow of the American Academy of Allergy, Asthma and Immunology (FAAAI), and Fellow of the National Academy of Inventors (FNI).

Dr. Nair and his colleagues discovered the suppressor factor in cancer serum (1978) and first reported that intravenous drug users manifest low natural killer cell activity (1986) and morphine induces apoptosis of normal lymphocytes (1997). In 1988, Dr. Nair reported for the first time (PNAS) that HIV recombinant purified gene product possesses significant biological activities. His original

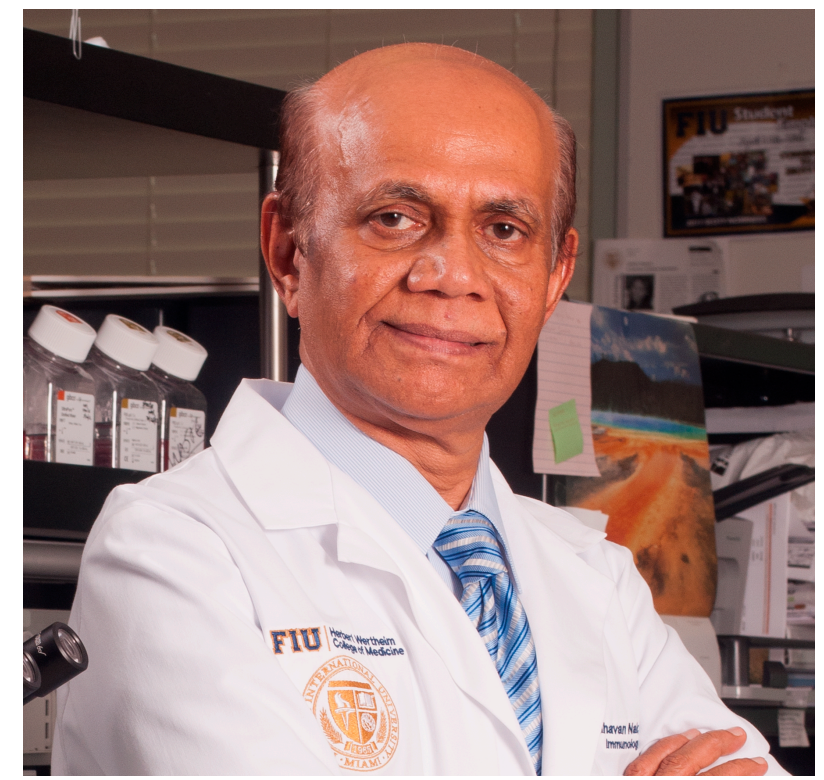

reports that cocaine increases the sensitivity to HIV infection by increasing the HIV coreceptors and methamphetamine exacerbates the HIV replication in dendritic cells had a profound effect on the role of these drugs on HIV disease progression. His recent research mainly involves the role of different drugs of abuse such as alcohol, morphine, cocaine, and methamphetamine on neuro-AIDS and therapeutic approaches to control neuro-AIDS by specific drug targeting to brain using nanotechnology.

Dr. Nair is the first FIU researcher to earn a prestigious MERIT Award from the National Institutes of Health recognizing outstanding competence and productivity in research (2008-2018). Dr. Nair is also the recipient of University of Michigan Distinguished Research Scientist Award (1990), Exceptional Research Scholar Award from State University of New York (2005), Excellence in Faculty Scholarship Award from FIU (2008), Presidential Leadership Operational Excellence Award from FIU in 2009, Wybran Award from Society of NeuroImmune Pharmacology (SNIP) in 2013, Distinguished Professorship from Florida State University system in 2013, and America's Super Professorship-Faculty Row Professor in 2014.

Dr. Nair has published more than 200 papers as first and/or senior author, mentored more than 50 
undergraduate, graduate, postdoctoral fellows, high school and minority students, served on various committees, organized various national and international conferences, chaired a number of scientific sessions, and served in various NIH study section committees as chair/member since 1980 . His research is currently supported by three major NIH grants. 